\title{
Chromosome analysis of epididymal and testicular spermatozoa in patients with azoospermia
}

\author{
Nunziatina Burrello ${ }^{1}$, Aldo E Calogero*, ${ }^{*}$, Adele De Palma ${ }^{1}$, Caterina Grazioso ${ }^{1}$, \\ Carlo Torrisi ${ }^{1}$, Nunziata Barone ${ }^{1}$, Carlo Pafumi ${ }^{2}$, Rosario D'Agata $^{1}$ and Enzo Vicari ${ }^{1}$
}

\author{
${ }^{1}$ Section of Endocrinology, Andrology and Internal Medicine, Department of Biomedical Sciences, and Master in \\ Andrological and Human Reproduction Sciences, University of Catania, Catania, Italy; ${ }^{2}$ Department of \\ Microbiology and Obstetrical Sciences, University of Catania, Catania, Italy
}

\begin{abstract}
Azoospermic patients can now father children once spermatozoa have been retrieved from the epididymis or the testis. However, there are concerns about the risk of chromosomal abnormalities since an increase in sperm aneuploidy rate has been reported in samples from patients with abnormal sperm parameters. The purpose of this study was therefore to evaluate the sperm aneuploidy and diploidy rates for chromosomes $8,12,18, X$ and $Y$ in spermatozoa extracted from the epididymes $(n=10)$ or the testes $(n=6)$ of patients with azoospermia. Ejaculated spermatozoa of healthy men $(n=14)$ served as control. Epididymal and testicular spermatozoa had an aneuploidy rate significantly higher than that found in ejaculated spermatozoa. The aneuploidy and diploidy rates of testicular spermatozoa were higher, but not significantly different, than those found in epididymal spermatozoa. This study has shown that azoospermic patients have an increased sperm aneuploidy rate. They should therefore be given appropriate genetic counselling before entering in-vitro fertilisation programs. European Journal of Human Genetics (2002) 10362 - 366. doi:10.1038/sj.ejhg.5200814
\end{abstract}

Keywords: sperm aneuploidy rate; azoospermia; epididymal sperm aspiration; testicular sperm aspiration; testicular sperm extraction; intracytoplasmic sperm injection

\section{Introduction}

Nowadays even with few spermatozoa extracted from the epididymis or the testis it is possible for patients with obstructive (OA) or non-obstructive azoospermia (NOA) to achieve pregnancy through intracytoplasmic sperm injection (ICSI). ${ }^{1}$ However, the use of this technique has raised a great deal of concern since it may increase the risk of transmitting genetic diseases to the offspring. Indeed, male infertility is associated with an increased frequency of abnormal caryotype ${ }^{2}$ which not only impairs spermatogenesis but results in the production of cytogenetically abnormal spermatozoa. Moreover, recent reports have shown that patients with abnormal sperm parameters, despite a normal blood karyotype, have an increased sperm aneuploidy rate which

\footnotetext{
*Correspondence: AE Calogero, Cattedra di Endocrinologia, Ospedale Garibaldi, Piazza S. Maria di Gesù, 95123 Catania, Italy.

Tel: +39-95-7592040; Fax: +39-95-310899; E-mail: acaloger@unict.it Received 29 January 2002; revised 26 March 2002; accepted 3 April 2002
}

correlates negatively with sperm density and with the percentage of normal forms. ${ }^{3-11}$ Therefore, there seems to be a direct relationship between spermatogenetic damage and sperm chromosome abnormalities. Since azoospermia represents the most severe degree of spermatogenetic impairment and given that mature germ cells can be retrieved from the epididymes or the testes of azoospermic patients and used to perform ICSI, we thought it was worth evaluating the aneuploidy rate of these cells. To accomplish this, we studied the aneuploidy rate of chromosomes 8,12 , $18, \mathrm{X}$ and $\mathrm{Y}$ by applying the multicolor fluorescence in-situ hybridisation (FISH) on spermatozoa retrieved from epididymes or the testes in a group of non-selected patients with OA or NOA undergoing an ICSI program.

Subjects and methods

Patient selection

Twenty-one unselected azoospermic patients, attending our Andrology and Reproductive Endocrinology Unit (AREU) for 
Table 1 Aneuploidy rates of spermatozoa extracted from the epididymis, the testis or the ejaculate

\begin{tabular}{|c|c|c|c|}
\hline & $\begin{array}{c}\text { Epididymal } \\
\text { spermatozoa } \\
\quad(\mathrm{n}=10)\end{array}$ & $\begin{array}{c}\text { Testicular } \\
\text { spermatozoa } \\
(\mathrm{n}=6)\end{array}$ & $\begin{array}{c}\text { Ejaculated } \\
\text { spermatozoa } \\
(\mathrm{n}=14)\end{array}$ \\
\hline Y-bearing spermatozoa (\%) & $47.22(39.56-50.4)^{*}$ & $47.19(43.75-62.5)^{*}$ & $49.86(48.07-51.81)$ \\
\hline XY disomy $(\%)$ & $0(0-1.39)$ & $0(0-0)$ & $0(0-0.17)$ \\
\hline XX disomy (\%) & $0.56(0.34-3.60)^{*}$ & $1.23(0.73-6.25)^{*} \dot{\dagger}$ & $0.20(0-0.51)$ \\
\hline Nullisomy rate & $0(0-0.37)$ & $0(0-1.02)$ & $0(0-0)$ \\
\hline \multicolumn{4}{|l|}{ Autosomes } \\
\hline Chromosome 8 disomy (\%) & $0.68(0-1.94)^{*}$ & $1.03(0-8.70)^{*}$ & $0.18(0.05-0.38)$ \\
\hline Chromosome 12 disomy (\%) & $0(0-0.17)$ & $0(0-0.48)$ & $0(0-0.20)$ \\
\hline Chromosome 18 disomy (\%) & $0.37(0-2.34)^{*}$ & $1.23(0.5-8.70)^{*}, \dagger$ & $0.20(0.05-0.45)$ \\
\hline Total disomy (\%) & $1.2(0-4.09)^{\star}$ & $2.23(0.96-17.4)^{*}$ & $0.46(0.15-0.65)$ \\
\hline
\end{tabular}

Results are expressed as medians with the range in parenthesis; ${ }^{\star} P<0.05$ vs Ejaculated spermatozoa, Mann-Whitney test; $\dagger P<0.05$ vs Epididymal spermatozoa, Mann-Whitney test.

their infertile status, were enrolled in this study. Spermatozoa were retrieved by epididymal sperm aspiration (PESA) from 10 patients with OA; eight of them had congenital bilateral absence of the vas deferens (CBAVD), one had acquired OA and one had post-vasectomy azoospermia. Testicular sperm aspiration (TESA) or testicular sperm extraction (TESE) was performed on 11 patients. No, or too few, spermatozoa were retrieved in five of them; consequently, these patients had to be excluded from the study because their testicular sperm aneuploidy rate could not be evaluated. Of the six patients studied, four had NOA, one had retrograde ejaculation as a result of pelvic lymphonode eradication for testicular teratocarcinoma, and one had acquired OA. PESA, TESA or TESE were performed under local anaesthesia by funicular infiltration, as previously reported. ${ }^{12}$ Fourteen healthy men with normal sperm density, motility and morphology, according the WHO criteria, ${ }^{13}$ served as controls. All patients and controls had a normal karyotype, evaluated by means of a standard cytogenetic technique. ${ }^{14}$ The protocol was approved by the local ethical committee. An informed written consent was obtained from each patient and control.

\section{Semen preparation for FISH analysis}

An aliquot of spermatozoa retrieved from the epididymis or the testis was washed three times in phosphate buffered saline $(\mathrm{pH} 7.2)$ and centrifuged at $650 \mathrm{~g}$ for $10 \mathrm{~min}$. The sediment was then fixed in methanol/acetic acid $(3: 1)$. The fixed specimens were stored at $-20^{\circ} \mathrm{C}$ until further processing. The sperm head decondensation was achieved by incubation with $25 \mathrm{mmol} / \mathrm{l}$ dithiothreitol, as previously reported. ${ }^{11}$ The treatment did not disrupt the sperm structure, including the tail, allowing an unequivocal differentiation between the spermatozoa and the other cells present in the ejaculate.

\section{DNA probes}

A double- and a triple-colour FISH were carried out on each patient and control, using alpha centromeric probes for chromosomes $8,12,18, \mathrm{X}$ and $\mathrm{Y}$. The probe mixture for triple FISH consisted of a repetitive DNA sequence of centromeric probes for chromosome X (pDMX1), labelled FITC, for chromosome Y (pLAY5.5) labelled Cy3 and for chromosome 12 (pBR12) labelled FITC and Cy3. The probe mixture for double-colour FISH consisted also of a repetitive DNA sequence of centromeric probes for chromosome 8 (pZ8.4) and for chromosome 18 (2Xba), labelled FITC or Cy3, respectively. The probes were provided by Prof M Rocchi, University of Bari (Bari, Italy).

\section{Hybridisation procedure}

Hybridisation was performed as previously reported. ${ }^{11}$ Briefly, each slide was denatured with a solution of $70 \%$ formamide $/ 2 \times \mathrm{SSC}(\mathrm{pH} 7.5)$ at $80^{\circ} \mathrm{C}$ for $150 \mathrm{~s}$. The slides were immersed in a 70, 90 and 100\% ethanol series for 3 min each and dried by air. The probes, precipitated and denatured at $80^{\circ} \mathrm{C}$ for $8 \mathrm{~min}$, were applied directly to the slides which were then covered with a coverslip and sealed with rubber cement. Hybridisation occurred overnight in a dark humidified container at $37^{\circ} \mathrm{C}$, after which the coverslip was removed and the slides were immersed three times in a posthybridisation wash of $50 \%$ formamide $/ 2 \times \mathrm{SSC}$ at $37^{\circ} \mathrm{C}$ for $5 \mathrm{~min}, 2 \times \mathrm{SSC}$ three times at $42^{\circ} \mathrm{C}$ for $5 \mathrm{~min}$ and $2 \times \mathrm{SSC} /$ $0.1 \%$ Tween 20 at room temperature for $5 \mathrm{~min}$. The slides were then mounted in 4',6-diamidino-2-pheneylindole 
(DAPI) counterstain and antifade and stored in the dark at $4{ }^{\circ} \mathrm{C}$ with a view to carrying out microscope observation. The slides were observed using an Axiophot fluorescence microscope (C. Zeiss, Oberkochen, Germany) with an appropriate set of single band filters for DAPI, FITC, and Cy3. Only intact spermatozoa bearing a similar degree of decondensation and clear hybridisation signals were scored; disrupted or overlapping spermatozoa were excluded from analysis. Spermatozoa were regarded as abnormal if they presented two (or more) distinct hybridisation signals for the same chromosome, each equal in intensity and size to the single signal found in normal monosomic nuclei. We considered only clear hybridisation signals, similar in size, separated from each other by at least one signal domain and clearly positioned within the sperm head. Divided (split) signals were not scored as disomies. Spermatozoa were scored as nullisomic if they showed no signal for a given chromosome, whereas the signal of the other chromosome tested was present. Finally, a spermatozoon was considered diploid in case it manifested two signals for each tested chromosome and in case the tail as well as the normal oval shape of a sperm head were evident. The absence of FISH signals in a spermatozoon head showing DAPI stain was considered a case of no-hybridisation. ${ }^{11}$

\section{Statistical analysis}

Results are shown as median and range throughout the study. The data were analysed using the Mann-Whitney test. SPSS 9.0 was used for statistical calculation. The coefficient of variation $(\mathrm{CV})$ of the total sperm aneuploidy rate was calculated by dividing the standard deviation by the mean and multiplying the result by 100 . A significant statistical difference was accepted when the $P$ value was lower than 0.05 .

\section{Results}

The median age of the patients who underwent PESA (32 years, range $28-41$ ) or TESA/TESE (37 years, range: $30-39$ ) was significantly higher $(P<0.05)$ than that of the controls (26 years, range 19-35). A total of 64092 spermatozoa was scored: 7060 epididymal spermatozoa (median 429, range 177 -1900), 2723 testicular spermatozoa (median 300, range 39-1314) and 54309 ejaculated spermatozoa (median 3930, range 3512 - 4018). The degree of sperm head decondensation and readability and the hybridisation efficiency rate (always greater than 99\%) of epididymal and testicular spermatozoa were similar to those obtained with ejaculated spermatozoa.

\section{Sex chromosome aneuploidy rate (Table 1)}

The percentage of X-bearing spermatozoa retrieved from the epididymes or the testes was not statistically different from that found in ejaculated spermatozoa, whereas the percentage of Y-bearing spermatozoa was significantly lower in both groups compared to that found in ejaculated spermatozoa
$(P<0.05)$. A very low XY disomy rate was found in all three groups of spermatozoa, suggesting a low rate of chromosome nondisjunction during the first meiotic division. On the other hand, significantly higher XX and YY disomy rates were found in epididymal and testicular spermatozoa compared to those in ejaculated spermatozoa $(P<0.05)$. The XX disomy rate found in testicular spermatozoa was significantly higher than that in epididymal spermatozoa $(P=0.03)$. Overall, the sex chromosome disomy rate was significantly higher in spermatozoa retrieved from the epididymes or the testes compared to that in ejaculated spermatozoa $(P<0.001$ for both groups). No statistical significant difference was found between the two groups of azoospermic patients. The sex chromosome nullisomy rate was very low and not statistically different in the three groups.

Chromosomes 8, 12 and 18 aneuploidy rate (Table 1) The chromosomes 8 and 18 disomy rates were significantly higher in epididymal and testicular spermatozoa compared to those in ejaculated spermatozoa $(P<0.05)$. The chromosome 18 disomy rate was higher in spermatozoa extracted from the testes with respect to that found in epididymal spermatozoa $(P=0.03)$. The chromosome 12 disomy rate was very low in all three groups. As against the controls, epididymal and testicular spermatozoa had a significantly higher disomy rate $(P<0.01)$. The testicular autosome disomy rate showed an upward trend compared to epididymal spermatozoa $(P=0.07)$. The nullisomy rates for the three autosomes studied were very low in all three groups.

\section{Total abnormal chromosome rate}

The total aneuploidy rate for the chromosome studied is reported in Figure 1. Epididymal and testicular spermatozoa had significantly higher sperm aneuploidy rates compared to ejaculated spermatozoa ( $P<0.001$ for both groups). The total aneuploidy rate of the azoospermic patients showed a marked inter-individual variation. All NOA patients had values substantially higher than the controls, whereas one patient of the epididymal group had values within the normal range and three had values close to the upper range observed in the control group. The median diploidy rate found in spermatozoa retrieved from the testis $(0.32 \%$, range: $0-1.95 \%)$ was higher than that found in epididymal $(0 \%$, range: $0-2.4 \%)$ or ejaculated spermatozoa $(0.04 \%$, range: $0-$ $0.20 \%)$. This difference, however, did not reach statistical significance.

\section{Discussion}

The FISH technique used to evaluate sperm chromosome abnormalities needs to score an adequate number of cells in order to give meaningful results. However, it is not always possible to retrieve a large number of spermatozoa from the epididymes or, which is more difficult, from the testes of 


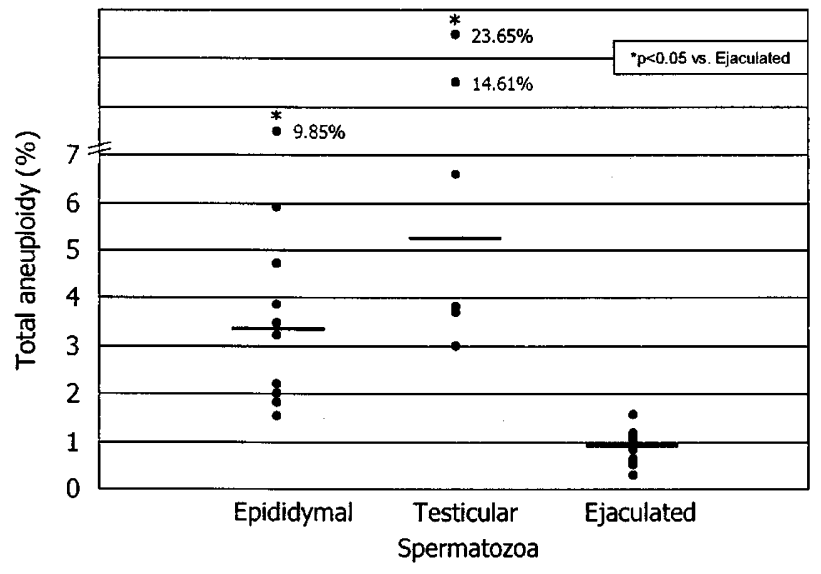

Figure 1 Individual aneuploidy rate for chromosomes 8, 12, $18, \mathrm{X}$ and $\mathrm{Y}$ in spermatozoa retrieved from the epididymis $(n=10)$ or the testis $(n=6)$ of patients with azoospermia. The aneuploidy rate found in ejaculated spermatozoa of 14 healthy men is shown as control.

azoospermic patients. Despite this limitation, it was worthwhile to carry out this study to evaluate the sperm aneuploidy rate in azoospermic patients requiring therapeutic PESA, TESA and/or TESE, given the paucity of substantial information on these patients and the frequent use of these spermatozoa in ICSI programs.

It is widely known that infertile patients have a higher frequency of constitutional chromosomal abnormalities which is more marked in patients with azoospermia. ${ }^{15,16}$ Even among infertile men with a normal blood karyotype, the frequency of chromosomal abnormalities restricted to the germ line is considerable. ${ }^{17,18}$ Furthermore, infertile men with impaired spermatogenesis may have an increased risk of producing aneuploid germ cells as a consequence of chromosomal pairing and/or disjunction errors during mitosis and/or meiosis ${ }^{15,19}$ which may constitute the underlying pathogenetic cause of the increased sperm aneuploidy rate found in patients with abnormal semen parameters. So it seems likely that patients with a more severe spermatogenetic impairment, such as those with NOA, are more prone to mitotic and/or meiotic errors during cell division and proliferation. ${ }^{9}$ This derangement in the meiotic process may be the consequence of a compromised testicular environment associated with impaired spermatogenesis, as shown in mice. ${ }^{20}$

This study has shown that patients with OA or NOA have a high rate of numerical chromosome abnormalities in spermatozoa extracted from the epididymis or the testis respectively, confirming recent preliminary results. ${ }^{7,21}$ Thus these data extend the high aneuploidy rate found in ejaculated spermatozoa of infertile men with various degree of semen abnormalities (which others and ourselves have demonstrated $^{3-11,}$ ) to spermatozoa extracted from the epididymes or the testes of patients with azoospermia.
The results of the present study are at variance with those of Martin and collaborators who evaluated the aneuploidy rate of testicular spermatozoa retrieved from three patients with NOA. ${ }^{22}$ Although these patients had an elevated disomy rate for chromosomes 13, 21 and XY compared to the controls, the difference did not reach statistical significance. This discrepancy may relate to the low number of patients studied or to the relatively low degree of testicular damage found in these three patients, as suggested by their normal serum FSH levels. Alternatively, it may indicate that the various possible causes of NOA have a different effect on the sperm aneuploidy rate, some disturbing the mechanisms of chromosome segregation during germ cell mitosis and/or meiosis to some extent and others only having minimal effects.

Both heterochromosomes and autosomes contributed to the increased aneuploidy rate found in both groups of azoospermic patients. Chromosome 12 showed the lowest aneuploidy rate and there was a very low frequency of nullisomic spermatozoa. This was similar to what we found in the ejaculated spermatozoa of patients with abnormal sperm parameters. ${ }^{11}$ Interestingly, azoospermic patients had a significantly lower number of spermatozoa bearing the $\mathrm{Y}$ chromosome, suggesting that a higher number of Yspermatozoa does not go through spermatogenesis. We have also observed a marked inter-individual variability in the aneuploidy rate among azoospermic patients. This variation was larger in testicular spermatozoa (CV: 89.6\%) compared to the spermatozoa retrieved from the epididymis (CV: $65.3 \%)$. This was predictable since patients with NOA are a more heterogeneous group with a severer spermatogenetic impairment and thus with a greater impact on chromosome segregation errors. Indeed, FSH and LH serum levels in the testicular group were significantly higher than those found in the epididymal one $(12.2 \pm 4.2 \mathrm{IU} / 1$ vs $3.6 \pm 0.4 \mathrm{IU} / 1$ and $10.0 \pm 1.9 \mathrm{IU} / \mathrm{l}$ vs $2.9 \pm 0.2 \mathrm{IU} / \mathrm{l}$, respectively). Since the use of testicular spermatozoa in ICSI programs leads to a lower ongoing pregnancy and delivery rates as a result of a higher abortion rate compared to epididymal or ejaculated spermatozoa, ${ }^{12,23}$ it can be hypothesised that this is an effect of a higher number of aneuploid spermatozoa found in these patients. ${ }^{24}$ The finding that also patients with OA have an increased sperm aneuploidy rate compared to controls suggests that a certain degree of spermatogenetic impairment exists also in these patients. ${ }^{25}$

In conclusion, this study has shown that spermatozoa retrieved from the epididymes or the testes of patients with azoospermia have an increased aneuploidy rate. This implies that whenever spermatozoa from patients with OA or NOA are used for ICSI may generate embryos with unbalanced genetic makeup and therefore an increased risk of chromosomal abnormality for the offspring. These patients should therefore be offered appropriate genetic counseling and preimplantation or prenatal diagnosis should be carefully considered. 


\section{Acknowledgments}

The authors thank David Farrugia, Lecturer, Faculty of Economics, University of Catania, for the English text editing. This study was supported in part by a grant from MURST (Cofinanziamento 1999), Rome, and from the University of Catania, Faculty of Medicine, Catania, Italy.

\section{References}

1 Devroey P, Liu J, Nagy Z, Tournaye H, Silber SJ, Van Steirteghem AC: Normal fertilization of human oocytes after testicular sperm extraction and intracytoplasmic sperm injection. Fertil Steril 1994; 62: 639-641.

2 Bourrouillou G, Dastugue N, Colombies N: Chromosome studies in 952 infertile males with a sperm count below 10 million/ml. Hum Genet 1985; 71: 366-367.

3 Moosani N, Pattinson HA, Carter MD, Cox DM, Rademaker AW, Martin RH: Chromosomal analysis of sperm from men with idiopathic infertility using sperm karyotyping and fluorescence in situ hybridization. Fertil Steril 1995; 64: 811-817.

4 McInnes B, Rademaker A, Greene CA, Ko E, Barclay L, Martin RH: Abnormalities for chromosomes 13 and 21 detected in spermatozoa from infertile men. Hum Reprod 1998; 13: $2787-$ 2790.

5 Pang MG, Hoegerman SF, Cuticchia AJ et al: Detection of aneuploidy for chromosomes $4,6,7,8,9,10,11,12,13,17$, $18,21, \mathrm{X}$ and $\mathrm{Y}$ by fluorescence in-situ hybridization in spermatozoa from nine patients with oligoasthenoteratozoospermia undergoing intracytoplasmic sperm injection. Hum Reprod 1999; 14: 1266-1273.

6 Rives N, Saint Clair A, Mazurier S et al: Relationship between clinical phenotype, semen parameters and aneuploidy frequency in sperm nuclei of 50 infertile males. Hum Genet 1999; 105: $266-272$.

7 Bernardini L, Gianaroli L, Fortini et al: Frequency of hyper-, hypohaploidy and diploidy in ejaculate, epididymal and testicular germ cells of infertile patients. Hum Reprod 2000; 15: $2165-2172$.

8 Nishikawa N, Murakami I, Ikuta K, Suzumori K: Sex chromosome analysis of spermatozoa from infertile men using fluorescence in-situ hybridization. J Assist Reprod Genet 2000; 17: $97-102$.

9 Ushijimal C, Kumasako Y, Kihaile PE, Hirotsuru K, Utsunomiya $\mathrm{T}$ : Analysis of chromosomal abnormalities in human spermatozoa using multi-colour fluorescence in-situ hybridization. Hum Reprod 2000; 15: 1107-1111.

10 Vegetti W, Van Assche E, Frias A et al: Correlation between semen parameters and sperm aneuploidy rates investigated by fluorescence in-situ hybridization in infertile men. Hum Reprod 2000; 15: $351-365$.
11 Calogero AE, De Palma A, Grazioso C et al: Aneuploidy rate in spermatozoa of selected men with abnormal semen parameters. Hum Reprod 2001; 16: 1172-1179.

12 Vicari E, Grazioso C, Burrello N, Cannizzaro M, D'Agata R, Calogero AE: Epididymal and testicular sperm retrieval in azoospermic patients and the outcome of intracytoplasmic sperm injection in relation to the etiology of azoospermia. Fertil Steril 2001; 75: $215-216$.

13 World Health Organization: WHO Manual for the Examination of Human Semen and Sperm-Cervical Mucus Interaction. Cambridge, University Press, Cambridge, 1992.

14 Rooney D, Czepulkowski B: Human Cytogenetics. A Practical Approach. Vol 1, Oxford University Press, Oxford, 1992.

15 Egozcue J, Templado C, Vidal F, Navarro J, Morer-Fargas F, Marina S: Meiotic studies in a series of 1100 infertile and sterile males. Hum Genet 1983; 65: $185-188$.

16 Luciani JM, Guichaoua MR, Delafontaine D, North MO, GabrielRobez O, Rumpler Y: Pachytene analysis in a 17;21 reciprocal translocation carrier: role of the acrocentric chromosomes in male sterility. Hum Genet 1987; 77: 246-250.

17 De Breekeleer M, Dao TN: Cytogenetic studies in male infertility: a review. Hum Reprod 1991; 6: 245-250.

18 Vendrell JM, Garcia F, Veiga A et alL: Meiotic abnormalities and spermatogenic parameters in severe oligoasthenozoospermia. Hum Reprod 1999; 14: 375 - 378.

19 Huang WJ, Lamb DJ, Kim ED et al: Germ-cell nondisjunction in testes biopsies of men idiopathic infertility. Am J Hum Genet 1999; 64: $1638-1645$.

20 Mroz K, Hassold JH, Hunt PA: Meiotic aneuploidy in the XXY mouse: evidence that a compromised testicular environment increases the incidence of meiotic errors. Hum Reprod 1998; 14: $1151-1156$.

21 Levron J, Aviram.Goldring A, Madgar I, Raviv G, Barkai G, Dor J: Sperm chromosome abnormalities in men with severe male factor infertility who are undergoing in vitro fertilization with intracytoplasmic sperm injection. Fertil Steril 2001, 76: 479484.

22 Martin RH, Greene C, Rademaker A, Barclay L, Ko E, Chernos J: Chromosome analysis of spermatozoa extracted from testes of men with non-obstructive azoospermia. Hum Reprod 2000; 15: $1121-1124$.

23 Palermo GD, Schlegel PN, Hariprashad JJ et al: Fertilization and pregnancy outcome with intracytoplasmic sperm injection for azoospermic men. Hum Reprod 1999; 14: $741-748$.

24 Rubio C, Gil-Salom M, Simon C et al: Incidence of sperm chromosomal abnormalities in a risk population: relationship with sperm quality and ICSI outcome. Hum Reprod 2001; 16: 2084-2092.

25 Meng MV, Black LD, Cha I et al: Impaired spermatogenesis in men with congenital absence of the vas deferens. Hum Reprod 2001; 16: 529-533. 